\title{
Spin Splitting in Symmetrical SiGe Quantum Wells
}

\author{
L.E. Golut* and E.L. Ivchenko \\ A.F. Ioffe Physico-Technical Institute, Russian Academy of Sciences, 194021 St. Petersburg, Russia
}

(Dated: November 21, 2018)

\begin{abstract}
Spin splitting of conduction electron states has been analyzed for all possible point symmetries of SiGe quantum well structures. A particular attention is paid to removal of spin degeneracy caused by the rotoinversion asymmetry of a (001) heterointerface between two diamond-lattice materials. The asymmetry is shown to result in spin splitting of both Rashba and Dresselhaus types in symmetrical SiGe quantum wells. Consequences of the spin splitting on spin relaxation are discussed.
\end{abstract}

Spin properties attract the great attention in recent years due to attempts to realize an electronic device based on the spin of carriers. Conduction electrons are obvious candidates for such devices, particularly in nanostructures where electron energy spectrum and shape of the envelope functions can be effectively engineered by the growth design, application of electric or magnetic fields as well as by illumination with light.

Various semiconductor materials are being involved in the spintronics activities. SiGe quantum well (QW) structures are among them.1,2,3,4 Although bulk Si and Ge have an inversion center, QW structures grown from these materials can lack such a center and allow the spin splitting of the electronic subbands.

The quantum engineering of spintronic devices is usually focused on the Rashba spin-dependent term to the electron effective Hamiltonian in heterostructures. This contribution appears due to asymmetry of the heteropotential (the so-called Structure Inversion Asymmetry, or SIA) and has no relation to the properties of a bulk semiconductor.

In III-V heterostructures, there exists another spindependent contribution called the Dresselhaus term that appears due to Bulk Inversion Asymmetry (BIA). It is commonly believed that the Dresselhaus contribution is absent in structures grown from centrosymmetric materials.

In the present work we show that the Dresselhaus-like spin splitting is possible in heterostructures made of Si and Ge due to the anisotropy of chemical bonds at interfaces.

Symmetry of a (001)-grown interface between $\mathrm{Si}_{1-x} \mathrm{Ge}_{x}$ and $\mathrm{Si}$ can be $\mathrm{C}_{2 \mathrm{v}}$ or $C_{4 \mathrm{v}}$ on average. $\frac{5}{.}$ The former point group describes the symmetry of an ideal heterointerface with the interfacial chemical bonds lying in the same plane. A nonideal interface containing monoatomic fluctuations has two kinds of flat areas with interfacial planes shifted with respect to each other by a quarter of the lattice constant. The local symmetry of each area is $\mathrm{C}_{2 \mathrm{v}}$ as well. However if the both kinds are equally distributed, the interface overall symmetry increases up to $C_{4 \mathrm{v}}$. It follows then that the symmetry of a $\mathrm{Si}_{1-x} \mathrm{Ge}_{x} / \mathrm{Si} \mathrm{QW}$ structure containing two interfaces is described by one of five point groups: $D_{2 \mathrm{~d}}$ or $D_{2 \mathrm{~h}}$ in case of two ideal interfaces with odd or even number of monolayers between them; $C_{2 \mathrm{v}}$ for a pair of ideal and rough interfaces; $C_{4 \mathrm{v}}$ or $D_{4 \mathrm{~h}}$ for two non-ideal interfaces of the overall symmetry $C_{4 \mathrm{v}}$ each, see Fig. 1.

Two of the above-mentioned groups, $D_{4 \mathrm{~h}}$ and $D_{2 \mathrm{~h}}$, contain the space inversion operation and forbid the spin splitting of electronic states. Three remaining groups allow the spin-dependent linear-in- $\boldsymbol{k}$ term $\mathcal{H}_{\boldsymbol{k}}=\gamma_{\alpha \beta} \sigma_{\alpha} k_{\beta}$. Here $\sigma_{\alpha}$ are the Pauli matrices and $\boldsymbol{k}=\left(k_{x}, k_{y}\right)$ is the in-plane electron wave vector.

In $\mathrm{Si}_{1-x} \mathrm{Ge}_{x} / \mathrm{Si}$ QWs grown along direction $z \|$ [001] with low enough content of Ge in the alloy layer, the lowest conduction band is located near the $X$ point of the Brillouin zone. Note that in the following we consider the electronic states attached to the $X_{z}$ valley because, due to the quantum confinement effect, its bottom lies lower than $X_{x}$ and $X_{y}$ valleys ${ }^{6}$ Hereafter the subscripts $x, y, z$ indicate three $X$ valleys as well as the Cartesian coordinate system with $x\|[100], y\|$ [010] being the in-plane axes. The symmetry analysis of the electron quantum confined states is based on the fact that at the $X$ point the bulk Bloch functions form projective representations of the point group $D_{4 \mathrm{~h}}$. All five above-mentioned groups are subgroups of $D_{4 \mathrm{~h}}$. In the group $D_{2 \mathrm{~d}}$, the Dresselhauslike term

$$
h_{D}(\boldsymbol{k})=\sigma_{x} k_{x}-\sigma_{y} k_{y}
$$

is an only invariant which can be constructed from the products $\sigma_{\alpha} k_{\beta}$. On the contrary, in the group $C_{4 \mathrm{v}}$, the only invariant combination is the Rashba term

$$
h_{R}(\boldsymbol{k})=\sigma_{x} k_{y}-\sigma_{y} k_{x} .
$$

The analysis shows that both combinations $h_{D}(\boldsymbol{k})$ and $h_{R}(\boldsymbol{k})$ are invariants of the group $C_{2 \mathrm{v}}$, i.e. both Dresselhaus- and Rashba-like spin-dependent terms are allowed.

In SiGe/Si QWs with high Ge content, the lowest conduction band may be located at the $L$ point. The bulk Bloch functions form projective representations of the $D_{3 \text { d }}$ point group which are $p$-equivalent to the usual representations of the same group. In this case the associated coordinate system $x^{\prime}, y^{\prime}, z^{\prime}$ is connected with the principal valley axis $z^{\prime} \|$ [111] and the in-plane axes $x^{\prime} \|[1 \overline{1} 0]$ (perpendicular to one of the mirror-reflection planes) and $y^{\prime} \|[11 \overline{2}]$. In (001)-grown QWs of the symmetry $D_{2 \mathrm{~h}}$ or $D_{4 \mathrm{~h}}$, intersection of these point groups with $D_{3 \mathrm{~d}}$ is $C_{2 \mathrm{~h}}$ with the inversion, so that the spin degeneracy is retained. On the other hand, the intersection of 

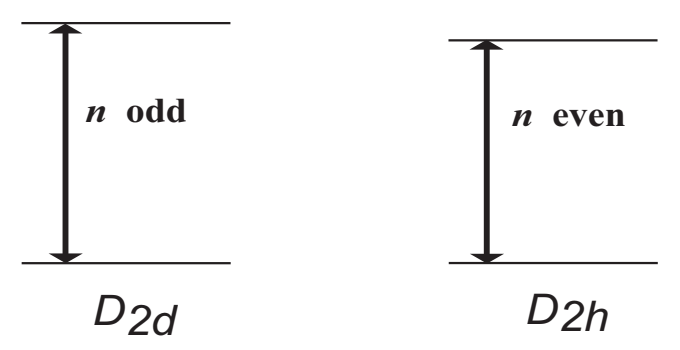

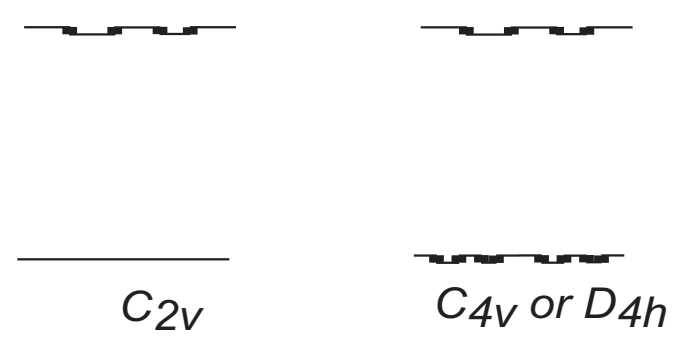

FIG. 1: Different interface profiles and QW point symmetries. The growth direction is [001], $n$ is a number of monoatomic layers.

the rest three groups with $D_{3 \mathrm{~d}}$ is $C_{\mathrm{s}}$. As a result, the effective Hamiltonian for the $L$-valley electrons in QWs of the symmetry $D_{2 \mathrm{~d}}, C_{2 \mathrm{v}}$ or $C_{4 \mathrm{v}}$ contains three linearly independent combinations $\sigma_{y^{\prime}} k_{x^{\prime}}, \sigma_{z^{\prime}} k_{x^{\prime}}$ and $\sigma_{x^{\prime}} k_{y^{\prime}}$ responsible for the spin splitting.

$\mathrm{SiGe} / \mathrm{Si}$ heterostructures grown in the (111)-direction have either $D_{3 \mathrm{~d}}$ or $C_{3 \mathrm{v}}$ point symmetry depending on the parity of monolayer numbers. The $D_{3 \mathrm{~d}}$ group has the inversion center and retains the spin degeneracy whereas the $C_{3 \mathrm{v}}$ group allows the spin splitting. The lowest conduction band in (111)-SiGe/Si QWs is located at the $L$ point of the Brillouin zone. Since $C_{3 \mathrm{v}}$ is a subgroup of $D_{3 \mathrm{~d}}$, the relevant symmetry is $C_{3 \mathrm{v}}$, and the invariant combination of the products $\sigma_{\alpha} k_{\beta}$ is the Rashba term

$$
\sigma_{x^{\prime}} k_{y^{\prime}}-\sigma_{y^{\prime}} k_{x^{\prime}}
$$

Microscopically, a linear-in- $\boldsymbol{k}$ correction to the conduction-band effective Hamiltonian is given by the second order of the perturbation theory

$$
\Delta \mathcal{H}=\frac{H_{\mathrm{SO}} H_{\boldsymbol{k} \boldsymbol{p}}^{\dagger}+H_{\boldsymbol{k} \boldsymbol{p}} H_{\mathrm{SO}}^{\dagger}}{E_{c}-E_{v}} .
$$

Here $H_{\boldsymbol{k} \boldsymbol{p}}$ and $H_{\mathrm{SO}}$ are interband $\boldsymbol{k} \cdot \boldsymbol{p}$ and spin-orbit interaction Hamiltonians, respectively. We take into consideration only the coupling of the conduction $X_{1}$ - and the valence $X_{4}$-states. The symmetry properties of the Bloch functions at the $X$ point of a diamond-lattice semiconductor crystal coincide with those of the following functions ${ }^{7}$

$$
\begin{gathered}
X_{1} \text {-states }:\left\{\begin{array}{l}
S=\cos (2 \pi z / a) \\
Z=\sin (2 \pi z / a)
\end{array},\right. \\
X_{4} \text {-states : }\left\{\begin{array}{l}
X=\sin (2 \pi x / a) \cos (2 \pi y / a) \\
Y=\cos (2 \pi x / a) \sin (2 \pi y / a)
\end{array}\right.
\end{gathered}
$$

where $a$ is the lattice constant. For the bulk states $X_{1}, X_{4}$ in the bases (2), (3), the interband matrix elements can be presented as

$$
H_{\boldsymbol{k} \boldsymbol{p}}=\mathcal{P}\left(\begin{array}{cc}
k_{x} & k_{y} \\
-k_{y} & -k_{x}
\end{array}\right), H_{\mathrm{SO}}=\mathcal{U}\left(\begin{array}{cc}
\sigma_{x} & -\sigma_{y} \\
-\sigma_{y} & \sigma_{x}
\end{array}\right) .
$$

Here $\mathcal{P}=\left(\hbar / m_{0}\right)\left\langle S\left|\hat{p}_{x}\right| X\right\rangle, \mathcal{U}=\left\langle S\left|U_{x}\right| X\right\rangle, \quad \hat{\boldsymbol{p}}$ is the momentum operator, and the pseudovector $\boldsymbol{U}=$ $\left(\hbar / 4 m_{0}^{2} c^{2}\right) \boldsymbol{\nabla} W \times \hat{\boldsymbol{p}}$ enters into the spin-orbit Hamiltonian $H_{\mathrm{SO}}=\boldsymbol{\sigma} \cdot \boldsymbol{U}$ ( $W$ is the microscopic potential).

The substitution of (4) into (11) results in

$$
\Delta \mathcal{H} \propto h_{D}(\boldsymbol{k})\left(\begin{array}{cc}
1 & 0 \\
0 & -1
\end{array}\right),
$$

where the second multiplier is a $2 \times 2$ matrix related to the basis (2). The matrix $\Delta \mathcal{H}$ does not lead to lifting the degeneracy of the $X_{1}$-states in the bulk centrosymmetric material, in accordance with the general symmetry consideration.

The splitting can be achieved if one takes into account the anisotropy of chemical bonds at the interfaces. It results in $\delta$-functional contributions to $H_{k p}$ and $H_{\mathrm{SO}}$ of the form

$$
\Delta H_{\boldsymbol{k} \boldsymbol{p}}=V_{\boldsymbol{k} \boldsymbol{p}} \delta\left(z-z_{i f}\right), \quad \Delta H_{\mathrm{SO}}=V_{\mathrm{SO}} \delta\left(z-z_{i f}\right),
$$

where $z_{i f}$ is the interface coordinate, and the matrices $V_{\boldsymbol{k} p}, V_{\mathrm{SO}}$ have few linearly independent components. In the case of the lowest symmetry under study, $C_{2 \mathrm{v}}$, each matrix is determined by four linearly-independent parameters

$$
\begin{aligned}
V_{\boldsymbol{k p}} & =\left(\begin{array}{cc}
P_{1} k_{x}+P_{2} k_{y} & P_{1} k_{y}+P_{2} k_{x} \\
P_{3} k_{y}+P_{4} k_{x} & P_{3} k_{x}+P_{4} k_{y}
\end{array}\right), \\
V_{\mathrm{SO}} & =\left(\begin{array}{cc}
U_{1} \sigma_{x}-U_{2} \sigma_{y} & -U_{1} \sigma_{y}+U_{2} \sigma_{x} \\
-U_{3} \sigma_{y}+U_{4} \sigma_{x} & U_{3} \sigma_{x}-U_{4} \sigma_{y}
\end{array}\right) .
\end{aligned}
$$

In the bases (22), (3), the parameters $P_{n}$ and $U_{n}(n=$ $1 \div 4)$ are purely imaginary.

By using Eqs. (1) and (5)-(7), one can show that, for a single interface of the $C_{2 \mathrm{v}}$-symmetry, the correction to the conduction-band Hamiltonian linear in $k_{x, y}$ and responsible for the removal of spin degeneracy is given by

$$
\Delta \mathcal{H}_{C_{2 \mathrm{v}}}=\left(\begin{array}{cc}
1 & 0 \\
0 & 1
\end{array}\right) H_{i f} \delta\left(z-z_{i f}\right) .
$$

Here $H_{i f}$ is a linear combination of the spin Pauli matrices that, in the first-order approximation in the perturbations (5), has the form

$$
\begin{aligned}
H_{i f} & =\frac{\mathcal{P}}{E_{0}}\left[\left(U_{3}-U_{1}\right) h_{D}(\boldsymbol{k})+\left(U_{4}-U_{2}\right) h_{R}(\boldsymbol{k})\right] \\
& -\frac{\mathcal{U}}{E_{0}}\left[\left(P_{1}+P_{3}\right) h_{D}(\boldsymbol{k})+\left(P_{2}+P_{4}\right) h_{R}(\boldsymbol{k})\right],
\end{aligned}
$$


with $E_{0}$ being the band gap between $X_{1}$ and $X_{4}$ states.

The electron effective Hamiltonian in an ideal QW contains a sum of two contributions (8) related to the left- $(l)$ and right-hand-side $(r)$ interfaces. If the $\mathrm{QW}$ contains an even number of monoatomic layers ( $D_{2 \mathrm{~h}}$ symmetry), then the corresponding parameters $U_{n}^{l}, U_{n}^{r}$ or $P_{n}^{l}, P_{n}^{r}$ are interconnected by

$$
\begin{gathered}
{\left[U_{1}, U_{2}, U_{3}, U_{4}\right]_{r}=\left[U_{3}, U_{4}, U_{1}, U_{2}\right]_{l},} \\
{\left[P_{1}, P_{2}, P_{3}, P_{4}\right]_{r}=\left[-P_{3},-P_{4},-P_{1},-P_{2}\right]_{l},}
\end{gathered}
$$

and if the QW contains an odd number of monoatomic layers $\left(D_{2 \mathrm{~d}}\right.$ symmetry), then

$$
\begin{aligned}
& {\left[U_{1}, U_{2}, U_{3}, U_{4}\right]_{r}=\left[U_{1},-U_{2}, U_{3},-U_{4}\right]_{l},} \\
& {\left[P_{1}, P_{2}, P_{3}, P_{4}\right]_{r}=\left[P_{1},-P_{2}, P_{3},-P_{4}\right]_{l} .}
\end{aligned}
$$

From here it readily follows that, in the former case, the electronic states in SiGe QWs are spin-degenerate $\left(H_{i f}=\right.$ 0 ) and, in the latter, the spin degeneracy is removed by a Dresselhaus-like linear-in- $\boldsymbol{k}$ terms. The similar analysis can be carried out for non-ideal SiGe QW structures of the $C_{2 \mathrm{v}}$ and $C_{4 \mathrm{v}}$ symmetry.

Spin splitting of L-electrons. Without spin, the basis functions for the $L$-point may be chosen as $s$-like for the $L_{1}$ conduction-band state (the Bloch function $S^{\prime}$ ), and as $p$-like $\left(X^{\prime}, Y^{\prime}\right)$ for the $L_{3^{\prime}}$ valence-band states. With spin, they are multiplied by the spin functions $\uparrow$ and $\downarrow$.

In a bulk diamond-lattice semiconductor, the spinorbit interaction results in a splitting by some value $\Delta$ of the $L_{3^{\prime}}$ valence band into the $\left(L_{4}^{-}, L_{5}^{-}\right)$and $L_{6}^{-}$subbands. In the three-fold degenerate $\Gamma$-point, the secondorder perturbation theory with allowance for $\Delta \neq 0$ gives rise to Dresselhaus-like conduction-band spin-splitting in

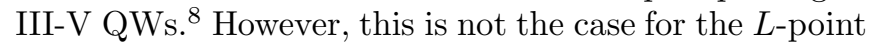
due to large energy spacing between the top valence band $L_{3^{\prime}}\left(X^{\prime}, Y^{\prime}\right)$ and lower lying valence-band states. Therefore, one should again take into account the interband spin-orbit interaction.

In the bulk, the spin-orbit coupling between the $L_{1}$ and $L_{3^{\prime}}$ bands is forbidden. However, the group $C_{\mathrm{s}}$ has only two symmetry operations: identical and reflection in the $\left(y^{\prime}, z^{\prime}\right)$-plane. The reflection yields $\left\langle S^{\prime}\left|U_{x^{\prime}}\right| X^{\prime}\right\rangle=$ $\left\langle S^{\prime}\left|U_{y^{\prime}, z^{\prime}}\right| Y^{\prime}\right\rangle=0$, but the following matrix elements are nonzero:

$$
\begin{aligned}
U_{1}^{\prime} & =\left\langle S^{\prime}\left|U_{y^{\prime}}\right| X^{\prime}\right\rangle, \\
U_{2}^{\prime} & =\left\langle S^{\prime}\left|U_{z^{\prime}}\right| X^{\prime}\right\rangle, \\
U_{3}^{\prime} & =\left\langle S^{\prime}\left|U_{x^{\prime}}\right| Y^{\prime}\right\rangle .
\end{aligned}
$$

Introducing the matrix element of the bulk $\boldsymbol{k} \cdot \boldsymbol{p}$ interaction $\mathcal{P}^{\prime}=\left(\hbar / m_{0}\right)\left\langle S^{\prime}\left|\hat{p}_{x}\right| X^{\prime}\right\rangle$, we obtain from Eqs. (1) and (10) for the $L$-states in a (001) QW

$\Delta \mathcal{H}_{C_{\mathrm{S}}}=-\frac{2 \mathcal{P}^{\prime}}{E_{0}^{\prime}} \delta\left(z-z_{i f}\right)\left(U_{2}^{\prime} \sigma_{z^{\prime}} k_{x^{\prime}}+U_{1}^{\prime} \sigma_{y^{\prime}} k_{x^{\prime}}+U_{3}^{\prime} \sigma_{x^{\prime}}\left\langle k_{y^{\prime}}\right\rangle\right)$.
Here $E_{0}^{\prime}$ is the energy gap between the $L_{1}$ and the $L_{3^{\prime}}$ states and the angular brackets mean averaging over the quantum-confined state. As a result the component $k_{y^{\prime}}=\left(k_{x}+k_{y}-2 k_{z}\right) / \sqrt{6}$ reduces to $\left\langle k_{y^{\prime}}\right\rangle=\left(k_{x}+k_{y}\right) / \sqrt{6}$ whereas the component $k_{x^{\prime}}=\left(k_{x}-k_{y}\right) / \sqrt{2}$ remains unchanged.

Inclusion of the spin splitting $\Delta$ of the valence band slightly modifies the coefficients in the above expression:

$$
\begin{aligned}
\Delta \mathcal{H}_{C_{\mathrm{S}}} & =-\delta\left(z-z_{i f}\right) \mathcal{P}^{\prime} \\
& \times\left[\left(\frac{1}{E_{0}^{\prime}-\Delta / 2}+\frac{1}{E_{0}^{\prime}+\Delta / 2}\right) U_{2}^{\prime} \sigma_{z^{\prime}} k_{x^{\prime}}\right. \\
& +\left(\frac{U_{1}^{\prime}+U_{3}^{\prime}}{E_{0}^{\prime}-\Delta / 2}+\frac{U_{3}^{\prime}-U_{1}^{\prime}}{E_{0}^{\prime}+\Delta / 2}\right) \sigma_{x^{\prime}}\left\langle k_{y^{\prime}}\right\rangle \\
& \left.+\left(\frac{U_{1}^{\prime}+U_{3}^{\prime}}{E_{0}^{\prime}-\Delta / 2}+\frac{U_{1}^{\prime}-U_{3}^{\prime}}{E_{0}^{\prime}+\Delta / 2}\right) \sigma_{y^{\prime}} k_{x^{\prime}}\right] .
\end{aligned}
$$

In (111)-grown QWs the relevant symmetry is $C_{3 \mathrm{v}}$ with the axis $C_{3}$ and two more mirror reflection planes in addition to the elements of $C_{\mathrm{s}}$ group. The rotation yields

$$
U_{1}^{\prime}=-U_{3}^{\prime}, \quad U_{2}^{\prime}=0,
$$

and we get from Eq. (11) the Rashba-like contribution for the (111) SiGe QWs with odd number of monolayers:

$$
\Delta \mathcal{H}_{C_{3 \mathrm{~V}}}=\delta\left(z^{\prime}-z_{i f}\right) \frac{2 \mathcal{P}^{\prime} U_{1}^{\prime}}{E_{0}^{\prime}}\left(\sigma_{x^{\prime}} k_{y^{\prime}}-\sigma_{y^{\prime}} k_{x^{\prime}}\right) .
$$

If the number of monolayers is even $\left(D_{3 \mathrm{~d}}\right.$ symmetry) then the inversion imposes the condition $U_{1}^{\prime}=0$, and spin splitting is absent.

Within the envelope function approximation, the electron wave function $\psi$ satisfies the Schrödinger equation with the effective Hamiltonian

$$
\mathcal{H}=\mathcal{H}_{0}\left(k_{z}, \boldsymbol{k}\right)+V(z)+\Delta \mathcal{H} .
$$

Here $\mathcal{H}_{0}$ is the bulk spin-independent Hamiltonian with $k_{z}=-\mathrm{i} \partial / \partial z, V(z)$ is the heteropotential, and the correction $\Delta \mathcal{H}$ is given by Eq. (8).

Instead of solving the Schrödinger equation with the Hamiltonian $\mathcal{H}$, one can equivalently find general solutions of the equations $\left(\mathcal{H}_{0}+V\right) \psi=E \psi$ within each homogeneous layer and then apply the boundary conditions

$$
\begin{aligned}
\psi\left(z_{i f}-0\right) & =\psi\left(z_{i f}+0\right) \\
\left.\left(v_{z} \psi\right)\right|_{z_{i f}-0} & =\left.\left(v_{z} \psi\right)\right|_{z_{i f}+0}+\frac{2 \mathrm{i}}{\hbar} H_{i f} \psi\left(z_{i f}\right),
\end{aligned}
$$

where the velocity operator $v_{z}=\hbar^{-1} \partial \mathcal{H}_{0} / \partial k_{z}$.

In order to estimate the spin splitting one needs to go beyond the envelope function approximation. This can be done in the pseudo-potential or tight-binding model which yields the matrix $H_{i f}$ in boundary conditions (13). The work on estimation of the interface-induced spin splitting in the microscopic tight-binding model is in progress. Here it suffices to note that the spin matrix 
$H_{i f}$ in Eq. (9) is non-vanishing if interatomic contributions to $H_{\mathrm{SO}}$ either $H_{\boldsymbol{k p}}$ are taken into account.

In this paper, we have analyzed spin splitting of electron states in SiGe heterostructures of all possible symmetries. The absence of inversion center can be also probed by means of second-harmonic generation experiments ${ }^{5,9.10}$ An ideal $\mathrm{Si}_{m} \mathrm{Ge}_{n}$ superlattice with odd $n$ and $m$ allows second-harmonic generation. It was demonstrated experimentally that $\mathrm{Si}_{m} \mathrm{Ge}_{n}$ superlattices with nominally odd and even $n, m$ possess comparable second-harmonic conversion efficiency. $\frac{9}{9}$ The weak nonlinear response can be explained by antiphase microscopic domains shifted with respect to each other by one monoatomic layer along the growth direction [001].

Similarly, different domains are characterized by opposite signs of the linear in $\boldsymbol{k}$ spin-dependent matrix $H_{i f}$ in Eq. (8). The influence of this kind of imperfection on the D'yakonov-Perel' spin relaxation of the conduction electrons depends on the relation between the linear dimension, $l_{D}$, of a single domain and the electron mean free path, $l$, in the interface plane. In the case $l_{D} \gg l$, the spin relaxation time of free carriers is the same as in a perfect structure:

$$
\frac{1}{\tau_{s}}=\frac{2}{\hbar^{2}}\left\langle\tau_{p} \operatorname{Tr}\left(H_{i f}^{2}\right)\right\rangle\left[\psi^{\dagger}\left(z_{i f}\right) \psi\left(z_{i f}\right)\right]^{2} .
$$

Here $\tau_{s}$ is the relaxation time of spin $z$-component (for in-plane spin the corresponding time is $2 \tau_{s}$ ). In Eq. (14) $\tau_{p}$ is the momentum scattering time, the angular brackets mean averaging over the carrier energy distribution, and $\psi\left(z_{i f}\right)$ is the interface value of the envelope function calculated at $H_{i f}=0$. In the opposite limiting case $l_{D} \ll l$, one has

$$
\frac{1}{\tau_{s}\left(l_{D}\right)} \approx \frac{1}{\tau_{s}} \frac{l_{D}}{l},
$$

where $\tau_{s}$ is given by Eq. (14). If the time $\tau_{p}$ is governed by scattering from the antiphase-domain walls (boundaries) one can use Eq. (14) for estimations of the spin relaxation times. Thus, we conclude that, even if the overall symmetry of a SiGe heterostructure is $D_{4 \mathrm{~h}}$ due to the antiphase domains, the lack of inversion center within a particular domain leads to the spin dephasing according to Eqs. (14) and (15). This opens a possibility to discuss the recent spin relaxation times measurements in these heterostructures, ${ }^{2,3}$

In conclusion, we have shown that linear-in- $\boldsymbol{k}$ spin splitting is present even in symmetrical SiGe QWs. It can be of Rashba, Dresselhaus, or both types. The splitting is caused by anisotropy of chemical bonds at interfaces.

This work is financially supported by the RFBR, INTAS, "Dynasty" Foundation - ICFPM, and by the Programmes of RAS and Russian Ministries of Science and Education.
* Electronic address: golub@coherent.ioffe.ru

1 S.D. Ganichev, U. Rössler, W. Prettl, E.L. Ivchenko, V.V. Bel'kov, R. Neumann, K. Brunner, and G. Abstreiter, Phys. Rev. B 66, 75328 (2002).

2 Z. Wilamowski, W. Jantsch, H. Malissa, and U. Rössler, Phys. Rev. B 66, 195315 (2002).

3 Z. Wilamowski and W. Jantsch, Phys. Rev. B, to be published.

4 E.Ya. Sherman, Phys. Rev. B 67, 161303 (2003).

5 X. Xiao, C. Zhang, A.B. Fedotov, Z. Chen, and M.M.T. Loy, J. Vac. Sci. Technol. B 15, 1112 (1997).

${ }^{6}$ V.Ya. Aleshkin and N.A. Bekin, J. Phys.: Condens. Matter
9, 4841 (1997).

7 Peter Y. Yu and Manuel Cardona, Fundamentals of Semiconductors. Physics and Material Properties, SpringerVerlag, Berlin, Heidelberg, 1996.

${ }^{8}$ U. Rössler and J. Kainz, Solid State Commun. 121, 313 (2002).

9 D.J. Bottomley, J.-M. Baribeau, H.M. van Driel, Phys. Rev. B 50, 8564 (1994).

10 G. Erley, R. Butz, and W. Daum, Phys. Rev. B 59, 2915 (1999). 